Technical Note

\title{
SIMU-RAD programme: a learning tool for radiation (photons and charged particles) interaction
}

\author{
Suffian MOHAMAD TAJUdIN ${ }^{1, a}$, Adila Hanim AMINORDIN SABRI ${ }^{1}$ \\ ${ }^{I}$ School of Medical Imaging, Faculty of Health Sciences, Universiti Sultan Zainal Abidin, Terengganu, Malaysia \\ ${ }^{a}$ E-mail address: suffian@unisza.edu.my
}

(received 4 February 2019; accepted 24 February 2019)

\begin{abstract}
Radiation education is necessary for a wide variety of people, such as radiation workers particularly for students of secondary school and higher education institution who learn radiation sciences. The fact that we could not see or feel radiation makes it difficult to understand it. The use of radiation trajectories shown on a personal computer should be useful to overcome this difficulty. In order to understand radiation behaviour inside the material, we have developed a Simu-Rad (Copyright: LY2018002738) by using Monte Carlo simulation programme. One who has no programming knowledge is able to simulate photons in a material through the developed programme. The program could become a computer aided learning tool for radiation related courses. We aim to facilitate lecturer from 'The Traditional Classroom' to 'The Flipped Classroom' for radiation education concerning in the era of IR 4.0. To validate our radiation simulator, we calculate photon linear attenuation coefficient $(\mu)$ of an aluminium material which commonly used as a filter in diagnostic radiology. $\mu$ is one of the main characteristics to understand how the radiation attenuated inside the materials. We calculate at energy photon of $662 \mathrm{keV}$ (Cs-137 radiation source) to compare our results of $\mu$ with the XCOM database. Consequently, the results from the developed simulator comparable with the database verified our programme to be used for radiation study.
\end{abstract}

Key words: Simu-Rad; Monte Carlo simulation; radiation education; linear attenuation coefficient $(\mu)$; XCOM database.

\section{Introduction}

\section{Monte Carlo Simulation}

There is a lot of work that has been done before by the scientist to simulate radiation using Monte Carlo methods. Monte Carlo method is a calculation using random numbers and statistical modeling to estimate mathematical functions and mimic the operations of complex systems [1,2]. In a radiation transport, calculation using the Monte Carlo method, the behavior of radiation inside material is followed by determining interaction points of radiation like photons and electrons, the type of interaction, scattering or absorption and else using random numbers [3]. The Monte Carlo is widely applied in medical physics to various problems such as an absorbed dose calculation to a cancer tumour and radiation shielding. The numbers of Monte Carlo simulation applications are increasing drastically with the improvements in the performance of computers [4,5].

Despite its useful benefit of performing radiation simulation, most of the available Monte Carlo methods are for experienced users and require a long learning time process. For the time being, Monte Carlo is the most accurate way of calculating radiation distribution dose in a patient. A number of Monte Carlo codes has been used to simulates how radiation enters the body, and more recently in the treatment planning for radiotherapy [6,7]. There are many Monte Carlo codes available such as Electron Gamma Shower (EGS), FLUKA, PENELOPPE, MCNP, GEANT4, XRAYSIM, and others to simulate radiation inside the materials [8]. Computer programming language skill is necessary to write the Monte Carlo simulation code before one is able to transport photon inside the materials and outputted correctly.

\section{EGS5}

EGS5 code has been developed at High Energy Accelerator Research Organization (KEK), Japan in 2005 [9]. EGS5 code is a general-purpose package for the Monte Carlo simulation to transport electrons and photons in an arbitrary geometry with energies above a few $\mathrm{keV}$ up to several hundred $\mathrm{GeV}$. In EGS5, the following interactions are considered:

(1) photons - photoelectric effect, Compton scattering, Rayleigh scattering, and pair production;

(2) electrons - Moller scattering, bremsstrahlung, multiple scattering, and continuous energy loss; 
(3) positrons - Bhabha scattering, bremsstrahlung, multiple scattering, continuous energy loss and annihilation in flight and at rest.

Simulation results obtained from this code were reported in many publications. As an example, simulations of the longitudinal and radial distributions of energy deposition of electrons of various energies made using EGS5 were compared with experimental results in the literature [10]. This example research confirms that the results from EGS5 simulation can be used for approximating the various energy depositions of electrons. Suffian et al. [11] had used EGS5 code to develop a photon field of $200 \mathrm{keV}$ to be used for detector calibration. Another study has indicated that EGS5 simulation results for energy range $662-1332 \mathrm{keV}$ with those obtained experimentally as well as by using other Monte Carlo codes MCNP and GEANT4 are in good agreement [12].

\section{Photon Attenuation}

The high energy photons can be dangerous for people who work in radiation field such as medical radiotherapy and particle accelerator laboratories. Radiation can cause cancer or radiation-related diseases. Thus, the proper radiation shielding with sufficient radiation attenuation is necessary to protect people and the environment from the harmful radiation. The basic ways to understand the behavior of photon interaction inside the materials is by measuring or calculate the linear attenuation coefficients $(\mu)$ of the particular material. To date, the $\mu$ values could be easily deduced from the XCOM database $[13,14]$ for low atomic number $(\mathrm{Z})$ to high $\mathrm{Z}$ number materials at any photon energies.

Many studies had been performed to measure and calculate $\mu$ values for shielding and soft tissue materials in order to get the value of discrepancies with the database [15-18]. It is an effective way to study the basic quantity such as photon attenuation $(\mu)$ for one to verify their measurements or simulations before apply to more complex geometry, like a real human body.

The photon attenuation is an intrinsic property of materials, which can be explained by the exponential decay equation [19] as:

$$
\frac{I}{I_{0}}=e^{-\mu x}
$$

where $I_{0}$ and $I$ are the initial and the attenuated intensity of photons, respectively. The quantity $x$ is the thickness of the material and $\mu$ is the linear attenuation coefficient. Commonly, the linear attenuation coefficient has a unit of $\mathrm{cm}^{-1}$. However, it is also usually identified as the mass attenuation coefficient $\mu / \rho$ in a unit of $\mathrm{cm}^{2} \mathrm{~g}^{-1}$. The attenuation of the photon in the material is determined by the interaction of photons with atoms, electrons or molecules in matter including Rayleigh scattering, Compton scattering, photoelectric effect, and pair production.

\section{SIMU-RAD (COPYRIGHT:LY2018002738)}

It seems now that in the near future, the Monte Carlo methodology will be widely used to study radiation properties, instead experiments. In this study, we present a Simu-Rad based on the EGS5 code for education purposes. Simu-Rad has been developed using PHP and Java applet to integrate and visualize the EGS5 Monte Carlo simulations engine into an easy to use web-based environment [20]. By integrating EGS5 code into our system, this system can be used for wide areas like radiation physics and shielding.

Through the Simu-Rad program, one could easily change the properties of radiation such as the types of radiation, its energy $(\mathrm{MeV})$ and the numbers of the incident photons to simulate from the selectable option in simulator interface (Figure 1).

\begin{tabular}{|c|c|c|}
\hline \multicolumn{3}{|l|}{ CLICK TO DISPLAY } \\
\hline Thickness (cm) & 10 & \\
\hline Material Type & Water $(\mathrm{H} 2 \mathrm{O})$ & $\boldsymbol{v}$ \\
\hline Particle Type & Photon V & \\
\hline Particle Energy in MeV & 0.25 & \\
\hline \multicolumn{2}{|c|}{ Number of Particle/Photons 20} & \\
\hline
\end{tabular}

Figure 1. User-friendly interface to perform radiation simulation with simple one "Generate" click to get the photon trajectories output.

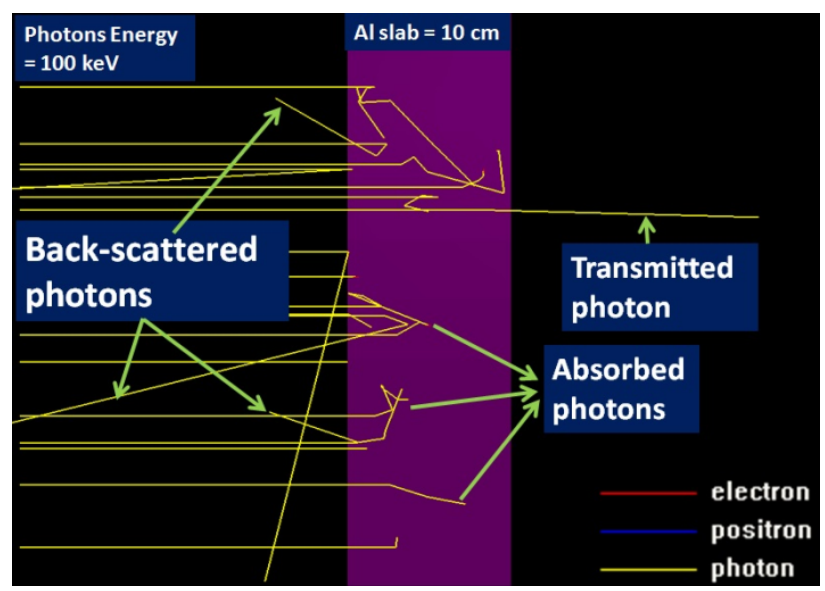

Figure 2. The output of Simu-Rad programme shows the photon trajectories inside the aluminum material by showing the types of photon interactions.

At this moment, FIVE (5) types of materials were prepared in the simulator as follows:

(1) Water $\left(\mathrm{H}_{2} \mathrm{O}\right)$ - for soft tissue water-equivalent;

(2) Poly methyl methacrylate (PMMA) - commonly used as phantom material;

(3) Lead $(\mathrm{Pb})$ - used for radiation shielding or collimator design;

(4) Concrete - used for radiation shielding such as radiation room design;

(5) Aluminum (Al) - commonly used as a radiation filter in diagnostic radiology. 
Despite its easier usage, we recommend to limit the number of incident photons for simplicity of manually eye evaluation at the outputted Simu-Rad programme of EGS5-CGview [21] to show photon trajectories for further analysis (Figure 2). It has to bear in mind the purpose of developed Simu-Rad is for education purpose only, where the statistic error will be large when only 50 numbers of photons simulated. In the real case of simulation, we normally simulated millions of photons for better statistics point of view.

In this study, the energy of the photons was set at $0.662 \mathrm{MeV}$ and was propagated in a parallel beam towards the aluminium material. We then determined the number of unscattered photons that pass through the aluminium manually by examined the photon trajectories as Figure 2. Therefore we limit the number of incident photons up to 50 photons for easier eye evaluation and short simulation running time. The same procedure was repeated for several thickness values of aluminium to create a graph of photon attenuation as a function of material thickness.

\section{Result and discussion}

A Simu-Rad used to calculate photon attenuation of 0.662 $\mathrm{MeV}$ through the aluminum material. The outputted photon trajectories were examined manually by eye to calculate the numbers of photons pass through the selected material. The photon trajectories were analyzed as TWO (2) following conditions:

(1) the number of unscattered photons passing through the material, and

(2) the number of transmitted photons (unscattered photons + scattered photons) passing through the material.

Figure 2 shows an example of photon interaction inside the aluminium of $10 \mathrm{~cm}$ thickness. Less photon were able to penetrate for the $10 \mathrm{~cm}$ case. Most of the photons were

(a)

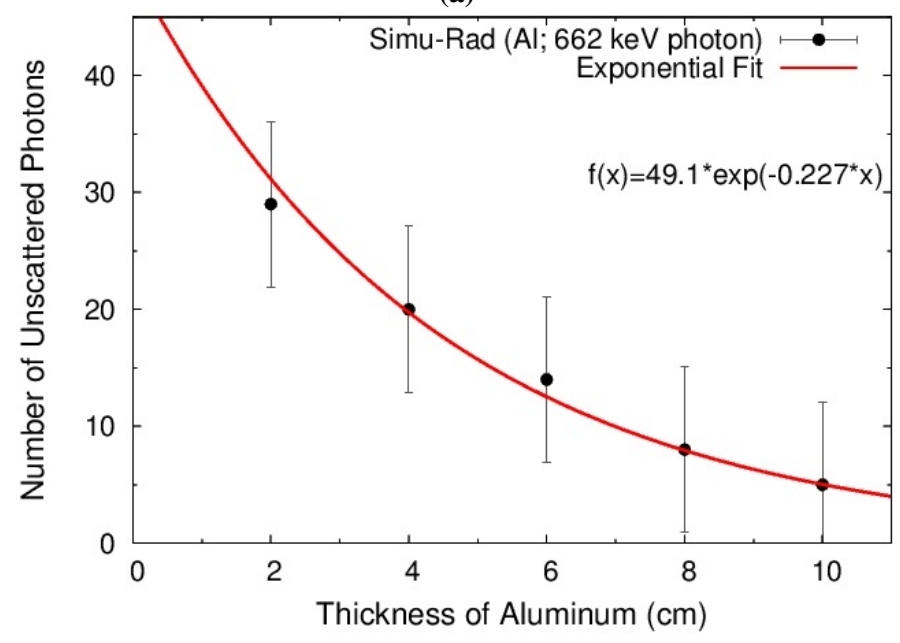

absorbed inside the material through 2 steps of photons interaction; Compton scattering either once or multiple scattering and followed by photoelectric absorption. Through the programme, one could consider how the main interaction varies depending on the photon energy and the material by key in or use the selectable option at the Simu-rad interface.

For the first case (1), the result obtained should be comparable with the XCOM database as any scattered photons were assumed to be absorbed by the material, while for the second case (2) represent the real cases as the photons may be scattered once (or multiple scattering) in the material before they pass through or exit the material. The uncertainty was evaluated as a square root of the simulated number of incident photons $(\sqrt{n})$.

The analysis of Simu-Rad simulation results in Figure 3a and $\mathbf{3 b}$ show they met the exponential decay equation as expected $[8,19]$. The linear attenuation coefficient values $(\mu)$ of aluminium at that particular energy were deduced from the exponential fitting. The Simu-Rad output the values of $\mu=$ $0.227 \mathrm{~cm}^{-1}$ for the first case and $\mu=0.119 \mathrm{~cm}^{-1}$ in the latter case. The error of $\mu$ values from exponential fitting for (a) and (b) is $4.9 \%$ and $4.4 \%$, respectively. The $\mu$ value in the later case lower than the first case as we could understand through the photon trajectories where some photons will undergo Compton scattering inside the material and exit the material rather than absorbed. The XCOM database value for $662 \mathrm{keV}$ photons is $2.07 \mathrm{~cm}^{-1}$. The disagreement was expected as the number of photons simulated in the program restricted to 50 photons to give poor statistics. The values of percentage deviation are still acceptable because they do not exceed than $10 \%(9.6 \%)$ for the first case. In order to reduce the error, the number of incident photons simulated needs to be increased, particularly to obtain reliable research data.

(b)

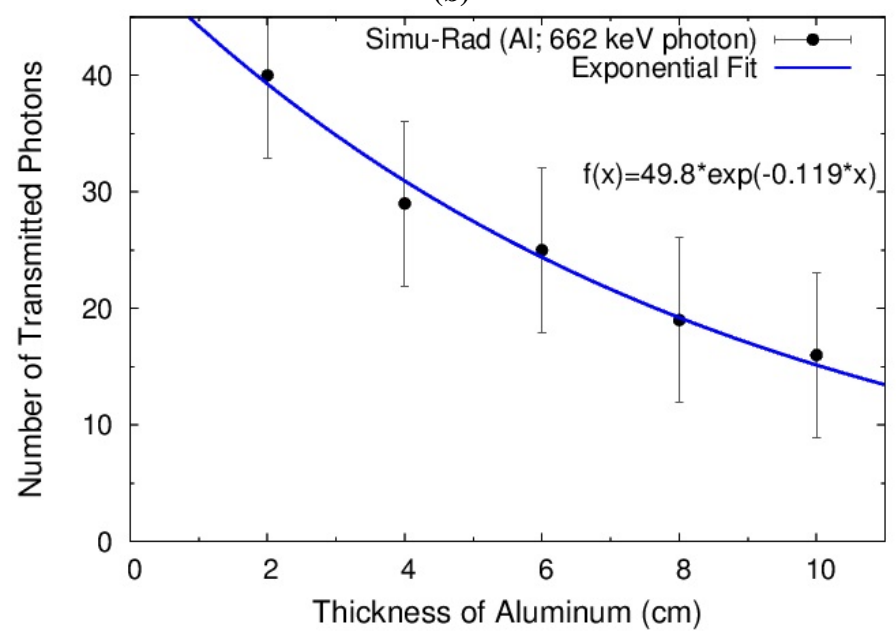

Figure 3. Simu-Rad result of $662 \mathrm{keV}$ photons for 50 numbers of incident photons strike the aluminium to give; $\left(\right.$ a) $\mu=0.227 \mathrm{~cm}^{-1}$ is for unscattered photons while (b) $\mu=0.119 \mathrm{~cm}^{-1}$ for transmitted photons. 


\section{Conclusion}

Simu-Rad (LY2018002738) was successfully developed to fulfill the growing demand to use technology in the students learning process, as well as to educate radiation technician and physicists. The simulator particularly can become computeraided learning tool as radiation education is necessary for a wide variety of people, such as radiation workers, students of radiography and medical imaging and others who work with radiation.

The simulated linear attenuation coefficients from the simulator are comparable to the values from XCOM database, where the deviations are about $10 \%$. Therefore, the developed simulator is a good educational tool where one could understand the type of photon interactions inside the materials and photon attenuation for shielding calculation. The program will benefit particularly for the institutions that have no irradiation facility or radiation sources.

\section{Acknowledgement}

We would like to thanks to our team members, Dr. Suhailan Safei and Dr. Azrul Amri Jamal from Faculty of Informatics and Computing, Universiti Sultan Zainal Abidin for supporting us all the way to accomplish this project.

\section{References}

[1] Harrison RL. Introduction to Monte Carlo Simulation. AIP Conf Proc. 2010;1204:17-21.

[2] Metropolis N, Ulam S. The Monte Carlo Method. J Am Stat Assoc. 1949;44(247)335-341.

[3] Hirayama H, Namito Y. Lecture Notes of Radiation Transport Calculation by Monte Carlo Method. KEK Internal.2001;2000-20.

[4] Dimov IT, Tonev OI. Monte Carlo Algorithms: Performance Analysis for Some Computer Architectures. J Comput Appl Math. 1993;48(3):253-277.

[5] Peter Ziegenhein, Pirner S, Ph Kamerling C, Oelfke U. Fast CPU-based Monte Carlo Simulation for Radiotherapy Dose Calculation. Phys Med Biol. 2015;60(15):6097-6111.

[6] Kara U, Tekin HO. Estımation of Absorbed Dose Distrıbution in Different Organs durıng the CT Scan: Monte Carlo Study. Austin J Radiol. 2017;4(1):1063.

[7] Larsson E, Ljungberg M, Strand SE, Jonsson BA. Monte Carlo Calculations of Absorbed Doses In Tumours using A Modified Moby Mouse Phantom for Pre-Clinical Dosimetry Studies. Acta Oncol. 2011;50:6,973-980.

[8] Sukara S, Rimjeam S. Simulation of Gamma Rays Attenuation Through Matters using the Monte Carlo Program. J Phys: Conf Ser. 2017;901:012141.

[9] Hirayama H, Namito Y, Bielajew AF, et al. The EGS5 Code System. 2005; SLAC-Report-730.

[10] Nelson WR, Field C. Comparison of EGS5 Simulations with Experiment. Nuclear Instrument and Methods in Physics Research. 2007;572(3):1083-1093.

[11] Tajudin SM, Namito Y, Sanami T, Hirayama H. Quasi-monoenergetic $200 \mathrm{keV}$ Photon Field using a Radioactive Source with Backscatter Layout. Japan J Appl Phys. 2014;53(11):116401.

[12] Malik AH, Shimazoe K, Takahashi H. EGS5 Simulations for the Development of Non-Contacting System to Online Measure the Radiotracer Concentration in Blood. Prog Nucl Sci Technol. 2014;4:290-293.

[13] Hubbell JH, Seltzer SM. X-Ray Mass Attenuation Coefficients. NISTIR 5632. https://www.nist.gov/pml/x-ray-mass-attenuationcoefficients.

[14] Berger MJ, Hubbell JH, Seltzer SM, et al. XCOM: Photon Cross Sections Database. NBSIR $87-3597$. http://www.nist.gov/pml/data/xcom/index.cfm.

[15] Pawar PP. Measurement of Mass and Linear Attenuation Coefficients of Gamma-Rays of AL for 514, 662 and 1280 keV Photons. J Chem Pharm Res. 2011;3(4): 899-903.

[16] Tekin HO, Manici T. Simulations of Mass Attenuation Coefficients for Shielding Materials using The MCNP-X code. Nucl Sci Techniques. 2017;28:95.

[17] Zhang L, Jia MC, Gong JJ, Xia WM. Simulation of Photon Attenuation Coefficients for High Effective Shielding Material LeadBoron Polyethyene. IOP Conf Ser: Earth Environ Sci. 2017; 100:012137.

[18] Ferreira CC, Ximenes RE, Garcia CAB, et al. Total Mass Attenuation Coefficient Evaluation of Ten Materials Commonly used to Simulate Human Tissue. J Phys: Conf Ser. 2010;249:012029.

[19] Knoll GF. Radiation Detection and Measurement. New York: John Wiley \& Sons, Inc. 2010.

[20] Suffian MT, Adila Hanim AS. Simu-Rad. Poster presented at Putra InnoCreative Carnival in Teaching and Learning (PicTL'18); 2018; Universiti Putra Malaysia, Malaysia.

[21] CGVIEW Particle Trajectory and Geometry Display Program. http://rcwww.kek.jp/research/egs/kek/cgview/ 